The prevalence of total malnutrition risk in this population, as determined by MUST, was lower at $12.1 \%$ compared to previously published smaller studies. 'At risk' groups were the young, the elderly and individuals of a white ethnic background.

Many patients 'triggered' with a MUST score of 1, BMI 18.5$20.0 \mathrm{~kg} / \mathrm{m}^{2}$, which is in fact within the normal range. This was particularly notable in a cohort of young women attending the Dermatology clinic.

The use of MUST in an outpatient setting requires further validation to ensure malnutrition risk is appropriately identified. Disclosure of Interest None Declared.

\section{PTH-135 OSTEOMYELITIS IN ADULT PATIENTS ON LONG-TERM PARENTERAL NUTRITION}

P Allan*, P Stevens, P Chadwick, A Teubner, A Abraham, G Carlson, S Lal. Intestinal Failure Unit, Salford Royal NHS Foundation Trust, Salford, UK

\subsection{6/gutjnl-2014-307263.581}

Introduction Osteomyelitis (OM) has rarely been reported in association with central venous catheter (CVC) use, but there are no reported data on the prevalence of $\mathrm{OM}$ in patients with intestinal failure (IF) with long term CVCs for parenteral nutrition (PN). We assessed period prevalence and characteristics of $\mathrm{OM}$ in adult patients on home PN.

Methods This was a retrospective study from a prospectively maintained database of patients referred to a national IF unit (IFU). Age, time on home PN, cultured organism (s) and $\mathrm{OM}$ site were recorded. Patients were divided into 2 groups: OM occurring in the context of acute (Type 2) IF (AIF) or chronic (Type 3) IF (CIF). Statistical analysis was Student's t-test.
Results 19 cases of OM occurred in 15 patients (6 male (40\%)) between 2004-2013. There were 9 cases of OM in 9 patients with AIF, and 10 cases in 6 patients with CIF; the latter yielded a period prevalence for $\mathrm{OM}$ of $1.3 \%$ when compared to the 457 home PN (HPN) patients managed by the IFU over this period. There were no cases of $\mathrm{OM}$ in the preceding 9 years (19952004) at the IFU. Median (range) age at commencing PN was 66 (30-72) years in AIF compared to 64 (29-70) years in CIF and mean $(95 \% \mathrm{CI})$ Charlson co-morbidity score was $3.7( \pm 2.5)$ in AIF compared to $2.2( \pm 0.9)$ in CIF. Patients with AIF had spent less time on PN before developing OM, compared to patients with CIF; despite this, the rate of CRBSIs was higher in the AIF than in the CIF group (see Table) as a result of patients with AIF contracting CRBSIs prior to specialised referral. Organisms and site of infection are shown in the table; identification of organism from the site of the OM successfully occurred in 3/ $9(33 \%)$ cases in AIF and 3/10 (30\%) cases in CIF, the remaining were identified via blood culture and aspiration of collections except one case where no organism was found, for which TB was suspected. All but one patient received at least 6 weeks antimicrobial chemotherapy; a further case required treatment for 3 months with antibiotics, 3 months with antifungals and 9 months with anti-TB medication. 4/10 (40\%) AIF cases required operative stabilisation: 3 spinal and 1 above knee amputation. 2/ $10(20 \%)$ CIF cases required operative stabilisation: 1 spinal and 1 pedal phalanx amputation. No patient died from OM.

Conclusion This is the first report of OM in a large cohort of patients with IF. While OM in IF is rare, the present reported experience from a national referral centre suggests that it may be increasing in incidence. IF practitioners should be vigilant for $\mathrm{OM}$ as a source of sepsis in this complex group of patients, since it carries significant morbidity.

Disclosure of Interest None Declared.

\begin{tabular}{|c|c|c|c|c|c|c|}
\hline & & \multicolumn{2}{|c|}{ Acute } & \multicolumn{2}{|c|}{ Chronic } & pralue \\
\hline \multicolumn{2}{|c|}{ Number of CRBSI (including diagnosis of OM) } & \multicolumn{2}{|c|}{7} & \multicolumn{2}{|c|}{13} & \\
\hline \multicolumn{2}{|c|}{ Total days on PN at time of diagnosis of OM } & \multicolumn{2}{|c|}{1782.0} & \multicolumn{2}{|c|}{28532.0} & \\
\hline \multicolumn{2}{|c|}{ CRBSI rate (per 1000 catheter days) } & \multicolumn{2}{|c|}{3.9} & \multicolumn{2}{|c|}{0.5} & \\
\hline \multirow{2}{*}{$\begin{array}{c}\text { Time from sepsis (CRBSI and } \\
\text { other) to diagnosis OM } \\
\text { (months) }\end{array}$} & Median & \multicolumn{2}{|c|}{2} & \multicolumn{2}{|c|}{1} & \multirow{2}{*}{0.476} \\
\hline & Range & 1 & 4 & 0 & 19 & \\
\hline \multirow{2}{*}{ om site } & Spinal & \multicolumn{2}{|c|}{7} & \multicolumn{2}{|c|}{6} & 0.434 \\
\hline & Extra-axial & \multicolumn{2}{|c|}{2} & \multicolumn{2}{|c|}{6} & 0.106 \\
\hline Micro-organisms identified & Staphylococcus & \multicolumn{2}{|c|}{3} & \multicolumn{2}{|c|}{7} & 0.081 \\
\hline & Coliforms & \multicolumn{2}{|c|}{2} & \multicolumn{2}{|c|}{8} & 0.005 \\
\hline & Fungi & \multicolumn{2}{|c|}{2} & \multicolumn{2}{|c|}{4} & 0.290 \\
\hline & Other & \multicolumn{2}{|c|}{4} & \multicolumn{2}{|c|}{8} & 0.111 \\
\hline
\end{tabular}




\section{PTH-136 A MYRIAD OF CAUSES FOR DIARRHOEA IN PATIENTS PRESENTING AT A GIANT (GI AND NUTRITION TEAM) CLINIC IN A CANCER CENTRE}

A Muls*, J Andreyev. GI Unit, Royal Marsden NHS Foundation Trust, London, UK

10.1136/gutjnl-2014-307263.582

Introduction Better cancer treatments have led to enormous improvements in the outcomes for these patients with the result that the overall number of survivors of cancer therapy continues to grow. However, after cancer treatment, up to $50 \%$ patients are left with diarrhoea - the most prevalent symptom. Causes are likely to encompass several contributing GI diagnoses.

Methods A service evaluation was conducted of new patients attending our clinic, reporting diarrhoea after treatment for cancer. All patients attending the clinic completed a patient recorded outcome measure describing their symptoms and a Bristol Stool Chart describing stool type. They were investigated using a peer reviewed investigational and management algorithm. Patient characteristics, symptom incidence and severity were recorded prospectively.

Results Over a 6 month period (July - Dec 2012), 207 patients were newly referred to the GIANTs. Of those, 104 (50\%) reported diarrhoea (type 6 or 7 Bristol Stool Chart). In this group there were slightly more men $(52 \%)$ than women (48\%). Their median age was 62 years (range: $22-89$ ). Primary tumour sites included urological cancer $(34 \%-82 \%$ of these prostate), gynaecological (22\%), colorectal (20\%), upper GI (10\%), haematological (8\%), and other (6\%). 69\% had undergone pelvic radiotherapy, $48 \%$ had been treated with surgery or received chemotherapy. $12 \%$ received pelvic radiotherapy alone, $6 \%$ surgery and $3 \%$ chemotherapy alone. Over a quarter (29\%) received all 3 treatment modalities.

Small intestinal bacterial overgrowth was found in $49 \%$. Bile acid malabsorption was newly diagnosed in 33\% of patients. Weak pelvic floor musculature was a contributing factor in $20 \%$. 13\% were diagnosed with new pancreatic insufficiency. Excess fibre intake $(>20 \mathrm{~g} /$ day $)$ was a contributory factor in $11 \%$ and Lansoprazole in up to $9 \%$ of patients. Other factors included: thyroid problems (9\%), anal fissure (5\%), rectal ulceration (5\%), faecal loading (5\%) and new onset Inflammatory Bowel Disease (3\%). A colorectal polyp was found in $16 \%$ of patients, 1 patient had a new colorectal cancer and 2 had a GI stricture.

$80 \%$ of patients had multiple causes for their diarrhoea. Most patients were discharged with a significant improvement in their symptoms with a median of 4 consultations (range 1-7) after systematic assessment and targeted management of the causes for their symptoms.

Conclusion Diarrhoea after cancer treatment is frequent in the patient cohort seen in our clinic. Several GI causes contribute to diarrhoea simultaneously in most patients but the majority can be discharged after a small number of consultations with a significant improvement or full resolution of their symptom if a systematic investigational and treatment approach is adopted.

Disclosure of Interest None Declared.

\section{PTH-137 THE GI AND NUTRITION TEAM SERVICE (GIANTS): MANAGING GI CONSEQUENCES OF CANCER TREATMENT - WHO? WHAT? AND HOW?}

A Muls*, J Andreyev. GI Unit, Royal Marsden NHS Foundation Trust, London, UK

10.1136/gutjnl-2014-307263.583
Introduction Our clinic specialising in gastrointestinal problems after cancer treatment, seems to be attracting patients with GI consequences resulting from a wider variety of cancer therapies. We present an analysis of new patients referred to our GI and Nutrition Team service.

Methods A service evaluation of new patients attending the clinic is ongoing after gaining appropriate approvals. All patients attending clinic complete a patient recorded outcome measure describing their symptoms, a Bristol Stool Chart describing stool type and a quality of life scale. Patients are investigated systematically depending on their symptoms, using a peer-reviewed algorithm. Patient characteristics, symptom incidence and severity are recorded prospectively.

Results Data for July-December 2012 were analysed. 207 patients were newly referred to the GIANTs. Their median age was 61.6 years (range: 22-89). 55\% were male. The largest group were patients treated for a urological malignancy (37\%), followed by those with a gynaecological (18\%), colorectal (16\%), upper GI cancer (12\%), other cancers (8\%), haematological malignancy (6\%) and no previous cancer diagnosis (3\%). $71 \%$ of patients had received pelvic radiotherapy, $3 \%$ chemotherapy, $11 \%$ GI surgery and $11 \%$ were treated with both chemotherapy and surgery. $4 \%$ had not yet received any cancer treatment and were usually referred to exclude the presence of IBD, a relative contra-indication to radiotherapy.

Comparing symptom profiles of patients who received pelvic radiotherapy $(\mathrm{n}=140)$ and those treated with other treatment modalities $(n=61)$, reveals that most patients were troubled by multiple symptoms: urgency (62 vs. 52\%), diarrhoea (57 vs. $51 \%$ ), tenesmus (47 vs. $43 \%$ ), flatulence (56 vs. $52 \%$ ), borborygmi (36\% vs $52 \%$ ) abdominal pain (39\% both groups), bloating (29 vs. $38 \%$ ), faecal leakage (16 vs. $31 \%)$ and nocturnal defaecation (31\% both groups). Rectal bleeding was reported by $34 \%$ of patients who received pelvic radiation, compared to $13 \%$ in the other group. Fatigue affected both groups (46 vs. 54\%).

The types of diagnosis to account for the symptoms made in both groups were similar: small intestinal bacterial overgrowth ( $24 \%$ pelvic radiotherapy vs. $28 \%$ other cancer treatments). Bile acid malabsorption was diagnosed in $16 \%$ (both groups) and pancreatic insufficiency in 6 and 5\%.

Conclusion Gastrointestinal problems after any cancer treatment are frequent and the symptom burden is high. The prevalence of symptoms patients describe after pelvic radiotherapy differs from those treated with other modalities but the causes identified for those symptoms are the same. A systematic management algorithm and multidisciplinary approach is required to manage those complex symptoms optimally.

Disclosure of Interest None Declared.

\section{PTH-138 TNF-A DEPENDENT ANGIOPOEITIN MEDIATED ANGIOGENESIS IN SPORADIC SMALL BOWEL ANGIODYSPLASIA; NOVEL PATHOPHYSIOLOGY AND POTENTIAL CLINICAL MARKER}

${ }^{1} G$ Holleran*, ${ }^{1 B}$ Hall, ${ }^{2} \mathrm{P}$ Crotty, ${ }^{3} \mathrm{~S}$ Smith, ${ }^{1} \mathrm{D}$ McNamara. 'Department of Clinical Medicine, Trinity College Dublin, Dublin, Ireland; ' ${ }^{2}$ Department of Histopathology, Tallaght Hospital, Tallaght, Ireland; ' ${ }^{3}$ Department of Clinical Medicine, Tallaght Hospital, Tallaght, Ireland

\subsection{6/gutjnl-2014-307263.584}

Introduction Angiodysplasias account for over 50\% of small bowel causes of obscure gastrointestinal bleeding. Angiodysplasias are thought to develop as a result of an imbalance in the 